\title{
Pulmonary fibrosis in the aftermath of the COVID-19 era (Review)
}

\author{
EIRINI VASARMIDI ${ }^{1}$, ELIZA TSITOURA $^{1}$, DEMETRIOS A. SPANDIDOS ${ }^{2}$, \\ NIKOLAOS TZANAKIS ${ }^{1}$ and KATERINA M. ANTONIOU ${ }^{1}$ \\ ${ }^{1}$ Department of Respiratory Medicine, University General Hospital of Heraklion, \\ Laboratory of Molecular and Cellular Pneumonology, Medical School, University of Crete; \\ ${ }^{2}$ Laboratory of Clinical Virology, School of Medicine, University of Crete, 71003 Heraklion, Greece
}

Received June 10, 2020; Accepted July 9, 2020

DOI: $10.3892 /$ etm. 2020.8980

\begin{abstract}
The year 2020 is characterized by the COVID-19 pandemic that has resulted in more than half a million deaths in recent months. The high mortality is associated with acute severe respiratory failure that results in ICU admission and intubation. While facing this fatal disease, research and clinical observations need to be carried out in order to evaluate the long-term effects of the COVID-19 acute respiratory distress syndrome (ARDS). Potent clinical and laboratory biomarkers should be studied to be able to predict the subgroup of patients that are going to deteriorate or develop lung fibrosis. The opportunity of personalized medicine is a good way to consider for these patients.
\end{abstract}

\section{Contents}

1. Introduction

2. Post-ARDS lung fibrosis

3. Possible biomarkers of disease progression

4. Is there a role for antifibrotic therapy?

5. Conclusion

\section{Introduction}

The novel coronavirus disease 2019 (COVID-19) pandemic, caused by severe acute respiratory syndrome coronavirus 2 (SARS-CoV-2), has generated huge concern for the high mortality rate and the lack of specific and effective treatment. Millions of people have been infected globally by SARS-CoV-2, which is a novel coronavirus, and the seventh member known

Correspondence to: Dr Katerina M. Antoniou, Department of Respiratory Medicine, University General Hospital of Heraklion, Laboratory of Molecular and Cellular Pneumonology, Medical School, University of Crete, 71003 Heraklion, Greece

E-mail:kantoniou@med.uoc.gr

Key words: SARS-CoV-2, COVID-19, lung, pulmonary fibrosis, interstitial disease to cause respiratory tract infections in humans. Four of them result in minor symptoms associated with the upper respiratory tract, while three coronaviruses, including SARS-CoV-2, are the causative agents of lower respiratory tract infections and major lung complications. The most critically ill patients in the context of SARS-CoV-2 infection, develop acute respiratory distress syndrome (ARDS) (1).

\section{Post-ARDS lung fibrosis}

Radiologically, most patients infected by SARS-COV-2 present with bilateral ground glass opacities with or without consolidation, and with preference of lower lobes (2). However, it should be considered that long-term lung impairment may develop following virus clearance, and in particular fibrotic interstitial lung disease. Pulmonary fibrosis can be idiopathic and considered as a genetically predisposed, age-related fibroproliferative disease, but chronic inflammation may also be involved in the pathogenesis of lung fibrosis. Importantly, pulmonary fibrosis is a recognised sequelae of ARDS, and several studies have shown that protective lung ventilation tends to diminish the radiographic abnormalities following ARDS (3).

The pathological correlate of ARDS is the diffuse alveolar damage (DAD) which is characterized by an initial (acute inflammatory) exudative phase with edema, hyaline membranes, and interstitial acute inflammation, followed by an organizing phase with loose organizing fibrosis mostly within the alveolar septa, and type II pneumocyte hyperplasia (4). A potent third and final stage of ARDS may be the fibrotic phase. Thille and coworkers described in a cohort of 159 autopsies from patients with ARDS, stating that these pathological findings can either resolve to normal lung parenchyma or progress to fibrosis (5). In this study, $4 \%$ of patients with a disease duration of less than 1 week, $24 \%$ of patients with a disease duration of between weeks 1 and 3, and $61 \%$ of patients with a disease duration of greater than 3 weeks, developed fibrosis (5). This description along with further data, support that pulmonary fibrosis begins early in the course of ARDS (3).

Abnormal immune mechanisms initiate and promote pulmonary fibrosis, possibly as a consequence of a cytokine storm. Dysregulated release of matrix metalloproteinases during the inflammatory phase of ARDS causes epithelial and 
endothelial injury. VEGF and cytokines such as IL-6 and TNFa are also implicated in the fibrotic process. It remains unclear why certain individuals are able to recover from such an insult, whereas others develop accumulation of fibroblasts and myofibroblasts and excessive deposition of collagen resulting in progressive pulmonary fibrosis (6). The crucial role of macrophages/monocytes, that can exert a proinflammatory or an anti-inflammatory effect based on the microenvironment in different stages, is also well established (7).

Available data indicate that about $40 \%$ of patients with COVID-19 develop ARDS, and 20\% of ARDS cases are severe (8). The prevalence of post-COVID-19 fibrosis will become apparent in time, but early analysis from patients with COVID-19 on hospital discharge suggests that more than a third of recovered patients develop fibrotic abnormalities. Additionally, $47 \%$ of patients had impaired Diffusing Capacity of the lungs for carbon monoxide (DLCO) and $25 \%$ had reduced total lung capacity (TLC). This seemed even worse in patients with severe disease (9). Long-term follow-up is definitely required to determine whether the reticulation represents irreversible fibrosis (10), although it seems that older patients with severe illness during treatment are more prone to develop fibrosis (11). Furthermore, the impact of COVID-19 in the progression of patients with preexisting interstitial lung disease still remains unknown and needs to be studied.

The two other strains of the coronavirus family that have been considered in previous years as highly pathogenic, SARS-CoV and MERS-CoV, are genetically similar to SARS-CoV-2 and cause contiguous lung disease. Data according to previous studies claim that there are reticular changes approximately two weeks after symptom onset in SARS-CoV that persist in half of the patients after one month (12). Longer follow-up data, 15 years later, showed that there were interstitial abnormalities in $4.6 \%$ of patients who had been infected with SARS-CoV (13). Although patients recovered from MERS are less well described in the literature, there is evidence of fibrotic abnormalities in about a third of patients in chest X-rays taken after hospital discharge (14).

Importantly, it has been shown that progressive pulmonary fibrosis can be a cause of mortality in a substantial proportion of patients with ARDS (15), while a considerable proportion of survivors will experience long-term impairment of lung function and radiographic abnormalities suggestive of pulmonary fibrosis $(16,17)$. The extent of reticulation on computed tomography correlates with quality of life and pulmonary function measures that indicate a restriction pattern, such as forced vital capacity (FVC) and DLCO (18).

Taking into consideration these data, even a relatively small degree of residual fibrosis could result in considerable morbidity and mortality in older patients who suffer from COVID-19, many of whom may already have lung disorders (19). Long-term follow-up studies are required to establish the true prevalence of post-COVID-19 fibrosis, but it is speculated that the consequences of COVID-19 could include a large cohort of patients with fibrosis and persistent or progressive lung impairment. Importantly, as thousands of individuals became affected by COVID-19, one can speculate that even rare complications will have major health effects at the population level.

\section{Possible biomarkers of disease progression}

The early identification of the subpopulation that is going to develop pulmonary fibrosis is of great importance. Based on the current knowledge, we assume that we have to act early in the ARDS course in order to avoid, delay or diminish the development of lung injury. At present, there are no reliable clinical or laboratory indicators early in disease progression to identify patients who are going to deteriorate and develop ARDS, although there are several markers associated to worse outcomes (1). Currently, it seems that apart from lung injury due to mechanical ventilation, lung damage is mostly a result of maladaptive immune responses leading to excessive cytokine release (19). The activation of lung-resident immune cells via pattern-recognition receptors, is followed by the release of high amounts of pro-inflammatory cytokines and extravasation of blood neutrophils and monocytes into the bronchi (20). Results from a recent Greek study reveal that patients with pneumonia caused by SARS-CoV-2, who developed severe respiratory failure, display hyper-inflammatory responses with features of either immune dysregulation or macrophage activation syndrome (MAS) (21). Immune dysregulation characterized by low expression of HLA-DR on CD14 monocytes, accompanied by profound depletion of CD4 lymphocytes, CD19 lymphocytes, and natural killer (NK) cells seem to predominate in most patients. The authors show that circulating monocytes are hyperactivated, producing IL- 6 and TNF- $\alpha$, possibly contributing to this immune dysregulation. Interestingly, all patients with SARS-CoV-2 and severe respiratory failure had either immune dysregulation or MAS, and this particular pattern is distinct when compared with patients suffered from bacterial or H1N1 associated pneumonia (21).

A study from Wuhan described the retrospective analysis of blood samples of 485 patients as an attempt to identify markers of mortality risk. Based on a mathematical modelling approach the authors identified three indicators (LDH, hs-CRP and lymphocytes) for COVID-19 prognostic prediction (22). The increase of LDH reflects tissue destruction and is regarded as a common sign of cell damage. In patients with severe pulmonary interstitial disease, the increase of LDH is significant and is one of the most important prognostic markers of lung injury (23). For critically ill patients with COVID-19, the rise in LDH level indicates an increase of the activity and extent of lung injury. The increase of hs-CRP, a well established marker for poor prognosis in sepsis and ARDS, reflects a persistent state of inflammation. Lymphopenia is a common feature in patients with COVID-19 and might be a critical factor associated with disease severity and mortality. Injured alveolar epithelial cells could induce the infiltration of lymphocytes, leading to persistent lymphopenia, as was seen in SARS-CoV-2 and MERS-CoV (22). Certainly, more studies need to be designed for the detection of patients at high risk for increased mortality, and for the development of fibrosis.

Previous studies in the literature have detected biomarkers of fibrogenesis in the bronchoalveolar fluid $24 \mathrm{~h}$ after the onset of ARDS that correlate with mortality. These include $\mathrm{N}$-terminal pro-peptide of type III collagen, C-terminal pro-peptide of type I collagen, TGF- $\beta$, and alveolar fibroblasts and fibrocytes (24-27). Fibrocytes have been shown to expand in the blood in the context of both physiologic wound repair 
and several diseases associated with fibrogenesis. As it is shown that the presence of fibrocytes in the BAL in ARDS correlates with poor outcomes (28), a recent study evaluated the expansion of fibrocytes in the bone marrow, blood, and lung of animal models of lung injury (29). Furthermore, a similar pattern in blood fibrocytes in patients with lung injury was described, the extent of which was predictive of outcomes (29), supporting the idea that fibrocytes could represent a useful biomarker.

\section{Is there a role for antifibrotic therapy?}

Currently, there are no approved therapies for any human coronavirus. Trials are based on drugs that are already approved for other diseases, have acceptable safety profiles or have been effective in animal studies against the other two highly pathogenic coronaviruses (30). Pirfenidone and nintedanib are the two approved antifibrotic drugs that, despite having different modes of action, are both effective in attenuating the rate of lung function decline and are widely considered to improve life expectancy $(31,32)$. Apart from the potent use of antivirals to reduce the viral effects, the use of antifibrotic therapies could also be under consideration based on the pulmonary fibrotic disease observed after COVID-19 recovery (33). Pirfenidone exerts anti-fibrotic, anti-oxidative and anti-inflammatory properties. Since there is no effective treatment strategy to reduce ARDS-associated lung injury and fibrosis, it could be suggested that pirfenidone could attenuate lung injury based on published data showing that pirfenidone reduces LPS-induced acute lung injury and subsequent fibrosis by suppressing NLRP3 inflammasome activation (34). Moreover, there is evidence of potential use of pirfenidone, azithromycin and prednisolone in the management of pulmonary fibrosis post-H1N1 ARDS, based on data from a case report of three patients (young males aged 40-59 years) (35). The current literature is suggestive that any potential antifibrotic intervention should be considered within the first week of ARDS onset so as to be more effective. This fact highlights even more the tremendous need for the identification of biomarkers early in the disease course to identify patients who are likely to progress to pulmonary fibrosis. Thus, the rationale for using antifibrotic therapy should be personalized and the role of precision medicine assumes prediction of high risk population, better understanding of pathophysiology and prevention of disease worsening or/and lung fibrosis development.

\section{Conclusion}

In recent months we all try to fight a new enemy, a novel coronavirus, that is almost unknown to the medical community. In parallel to the efforts regarding the pathophysiology of this new disease, we also need to evaluate the proportion of patients that are going to develop chronic lung disease following COVID-19 recovery. Certainly, more attention should be given to new studies that will focus on revealing potent predictors of mortality in these patients, and discovering biomarkers of progression of COVID-ARDS to pulmonary fibrosis. Until the discovery of an effective vaccine, apart from protective lung ventilation, a close follow-up of patients recovered from COVID-19-ARDS is highly recommended.

\section{Acknowledgements}

Not applicable.

\section{Funding}

No funding was received.

\section{Availability of data and materials}

Not applicable.

\section{Authors' contributions}

EV, ET, DAS, NT and KA each substantially contributed to the writing and revision of the manuscript, read and approved the final version.

\section{Ethics approval and consent to participate}

Not applicable.

\section{Patient consent for publication}

Not applicable.

\section{Competing interests}

DAS is the Editor-in-Chief for the journal, but had no personal involvement in the reviewing process, or any influence in terms of adjudicating on the final decision, for this article. The other authors declare that they have no competing interests.

\section{References}

1. Guan WJ, Ni ZY, Hu Y, Liang WH, Ou CQ, He JX, Liu L, Shan H, Lei CL, Hui DSC, et al; China medical treatment expert group for Covid-19: Clinical characteristics of coronavirus disease 2019 in China. N Engl J Med 382: 1708-1720, 2020.

2. Wang D, Hu B, Hu C, Zhu F, Liu X, Zhang J, Wang B, Xiang H, Cheng Z, Xiong Y, et al: Clinical characteristics of 138 hospitalized patients with 2019 novel coronavirus-infected pneumonia in Wuhan, China. JAMA 323: 1061, 2020.

3. Burnham EL, Janssen WJ, Riches DWH, Moss M and Downey GP: The fibroproliferative response in acute respiratory distress syndrome: Mechanisms and clinical significance. Eur Respir J 43: 276-285, 2014.

4. Cardinal-Fernández P, Lorente JA, Ballén-Barragán A and Matute-Bello G: Acute respiratory distress syndrome and diffuse alveolar damage. New insights on a complex relationship. Ann Am Thorac Soc 14: 844-850, 2017

5. Thille AW, Esteban A, Fernández-Segoviano P, Rodriguez JM, Aramburu JA, Vargas-Errázuriz P, Martín-Pellicer A, Lorente JA and Frutos-Vivar F: Chronology of histological lesions in acute respiratory distress syndrome with diffuse alveolar damage: A prospective cohort study of clinical autopsies. Lancet Respir Med 1: 395-401, 2013

6. George PM, Wells AU and Jenkins RG: Pulmonary fibrosis and COVID-19: the potential role for antifibrotic therapy. Lancet Respir Med: https://doi.org/10.1016/S2213-2600(20)30225-3.

7. Huang X, Xiu H, Zhang S and Zhang G: The role of macrophages in the pathogenesis of ALI/ARDS. Mediators Inflamm 2018: $1264913,2018$.

8. Wu C, Chen X, Cai Y, Xia J, Zhou X, Xu S, Huang H, Zhang L, Zhou X, Du C, et al: Risk factors associated with acute respiratory distress syndrome and death in patients with coronavirus disease 2019 pneumonia in Wuhan, China. JAMA Intern Med: doi:10.1001/jamainternmed.2020.0994. 
9. Mo X, Jian W, Su Z, Chen M, Peng H, Peng P, Lei C, Chen R, Zhong N and Li S: Abnormal pulmonary function in COVID-19 patients at time of hospital discharge. Eur Respir J 55: 2001217 , 2020.

10. Hu Q, Guan H, Sun Z, Huang L, Chen C, Ai T, Pan Y and Xia L: Early CT features and temporal lung changes in COVID-19 pneumonia in Wuhan, China. Eur J Radiol 128: 109017, 2020.

11. Wei J, Yang H, Lei P, Fan B, Qiu Y, Zeng B, Yu P, Lv J, Jian Y and Wan C: Analysis of thin-section CT in patients with coronavirus disease (COVID-19) after hospital discharge. J XRay Sci Technol 28: 383-389, 2020

12. Ooi GC, Khong PL, Müller NL, Yiu WC, Zhou LJ, Ho JC, Lam B, Nicolaou S and Tsang KW: Severe acute respiratory syndrome: Temporal lung changes at thin-section CT in 30 patients. Radiology 230: 836-844, 2004

13. Zhang P, Li J, Liu H, Han N, Ju J, Kou Y, Chen L, Jiang M, Pan F, Zheng Y, et al: Long-term bone and lung consequences associated with hospital-acquired severe acute respiratory syndrome: A 15-year follow-up from a prospective cohort study. Bone Res 8: 8, 2020.

14. Das KM, Lee EY, Singh R, Enani MA, Al Dossari K, Van Gorkom K, Larsson SG and Langer RD: Follow-up chest radiographic findings in patients with MERS-CoV after recovery. Indian J Radiol Imaging 27: 342-349, 2017.

15. Meduri GU, Headley S, Kohler G, Stentz F, Tolley E, Umberger R and Leeper K: Persistent elevation of inflammatory cytokines predicts a poor outcome in ARDS. Plasma IL-1 beta and IL-6 levels are consistent and efficient predictors of outcome over time. Chest 107: 1062-1073, 1995.

16. Masclans JR, Roca O, Muñoz X, Pallisa E, Torres F, Rello J and Morell F: Quality of life, pulmonary function, and tomographic scan abnormalities after ARDS. Chest 139: 1340-1346, 2011.

17. Desai SR, Wells AU, Rubens MB, Evans TW and Hansell DM: Acute respiratory distress syndrome: $\mathrm{CT}$ abnormalities at long-term follow-up. Radiology 210: 29-35, 1999.

18. Burnham EL, Hyzy RC, Paine R III, Coley C II, Kelly AM, Quint LE, Lynch D, Janssen WJ, Moss M and Standiford TJ: Chest CT features are associated with poorer quality of life in acute lung injury survivors. Crit Care Med 41: 445-456, 2013.

19. Spagnolo P, Balestro E, Aliberti S, Cocconcelli E, Biondini D, Casa GD, Sverzellati N and Maher TM: Pulmonary fibrosis secondary to COVID-19: A call to arms? Lancet Respir Med: May 15, 2020 (Epub ahead of print).

20. Risitano AM, Mastellos DC, Huber-Lang M, Yancopoulou D, Garlanda C, Ciceri F and Lambris JD: Complement as a target in COVID-19? Nat Rev Immunol 20: 343-344, 2020.

21. Giamarellos-Bourboulis EJ, Netea MG, Rovina N, Akinosoglou K, Antoniadou A, Antonakos N, Damoraki G, Gkavogianni T, Adami ME, Katsaounou P, et al: Complex immune dysregulation in COVID-19 patients with severe respiratory failure. Cell Host Microbe 27: 992-1000.e3, 2020.

22. Yan L, Zhang H-T, Goncalves J, Xiao Y, Wang M, Guo Y, Sun C, Tang X, Jing L, Zhang M, et al: An interpretable mortality prediction model for COVID-19 patients. Nat Mach Intell 2: 283-288, 2020

23. Kishaba T, Tamaki H, Shimaoka Y, Fukuyama $H$ and Yamashiro S: Staging of acute exacerbation in patients with idiopathic pulmonary fibrosis. Lung 192: 141-149, 2014.
24. Wang Y, Wang H, Zhang C, Zhang C, Yang H, Gao R and Tong Z: Lung fluid biomarkers for acute respiratory distress syndrome: A systematic review and meta-analysis. Crit Care 23: 43, 2019.

25. Capelozzi VL, Allen TC, Beasley MB, Cagle PT, Guinee D, Hariri LP, Husain AN, Jain D, Lantuejoul S, Larsen BT, et al: Molecular and Immune Biomarkers in Acute Respiratory Distress Syndrome: A Perspective From Members of the Pulmonary Pathology Society. Arch Pathol Lab Med 141: 1719-1727, 2017.

26. Madtes DK, Rubenfeld G, Klima LD, Milberg JA, Steinberg KP, Martin TR, Raghu G, Hudson LD and Clark JG: Elevated transforming growth factor- $\alpha$ levels in bronchoalveolar lavage fluid of patients with acute respiratory distress syndrome. Am J Respir Crit Care Med 158: 424-430, 1998.

27. Forel JM, Guervilly C, Hraiech S, Voillet F, Thomas G, Somma C, Secq V, Farnarier C, Payan MJ, Donati SY, et al: Type III procollagen is a reliable marker of ARDS-associated lung fibroproliferation. Intensive Care Med 41: 1-11, 2015.

28. Quesnel C, Piednoir P, Gelly J, Nardelli L, Garnier M, Leçon V, Lasocki S, Bouadma L, Philip I, Elbim C, et al: Alveolar fibrocyte percentage is an independent predictor of poor outcome in patients with acute lung injury. Crit Care Med 40: 21-28, 2012.

29. Lin CM, Alrbiaan A, Odackal J, Zhang Z, Scindia Y, Sung SJ, Burdick MD and Mehrad B: Circulating fibrocytes traffic to the lung in murine acute lung injury and predict outcomes in human acute respiratory distress syndrome: A pilot study. Mol Med 26: $52,2020$.

30. Tsitoura E, Bibaki E, Bolaki M, Vasarmidi E, Trachalaki A, Symvoulakis EK, Spandidos DA and Antoniou MK: [Comment] Treatment strategies to fight the new coronavirus SARS-CoV-2: A challenge for a Rubik's Cube solver. Exp Ther Med 20: 147-150, 2020.

31. Margaritopoulos GA, Trachalaki A, Wells AU, Vasarmidi E, Bibaki E, Papastratigakis G, Detorakis S, Tzanakis N and Antoniou KM: Pirfenidone improves survival in IPF: Results from a real-life study. BMC Pulm Med 18: 177, 2018.

32. Antoniou K, Markopoulou K, Tzouvelekis A, Trachalaki A, Vasarmidi E, Organtzis J, Tzilas V, Bouros E, Kounti G, Rampiadou $\mathrm{C}$, et al: Efficacy and safety of nintedanib in a Greek multicentre idiopathic pulmonary fibrosis registry: A retrospective, observational, cohort study. ERJ Open Res 6: 00172-02019, 2020.

33. Shi H, Han X, Jiang N, Cao Y, Alwalid O, Gu J, Fan Y and Zheng C: Radiological findings from 81 patients with COVID-19 pneumonia in Wuhan, China: A descriptive study. Lancet Infect Dis 20: 425-434, 2020.

34. Li Y, Li H, Liu S, Pan P, Su X, Tan H, Wu D, Zhang L, Song C, Dai M, et al: Pirfenidone ameliorates lipopolysaccharide-induced pulmonary inflammation and fibrosis by blocking NLRP3 inflammasome activation. Mol Immunol 99: 134-144, 2018.

35. Saha A, Vaidya PJ, Chavhan VB, Achlerkar A, Leuppi JD and Chhajed PN: Combined pirfenidone, azithromycin and prednisolone in post-H1N1 ARDS pulmonary fibrosis. Sarcoidosis Vasc Diffuse Lung Dis 35: 85-90, 2018.

This work is licensed under a Creative Commons Attribution-NonCommercial-NoDerivatives 4.0 International (CC BY-NC-ND 4.0) License. 